\title{
Editorial: Effect of cyclosporin A on the survival of corneal grafts in rabbits
}

The substantially improved prognosis for corneal grafts which has resulted from better surgical techniques and applied corneal biology have made the allograft reaction the most important single cause of corneal graft failure and a major obstacle to progress in corneal transplantation. Although allograft reactions are rare in avascular corneas, in grossly diseased vascularised corneas graft rejection is a significant problem. For example, it occurs in over one-half of penetrating grafts performed in eyes with herpes simplex keratitis.

The cornea is unique in that clinical manifestations of graft reactions can be observed easily, and topically administered corticosteroids are frequently effective in preventing and suppressing allograft reactions. However, corticosteroids are by no means always effective, and their use is associated with serious complications-in particular, the development of glaucoma and cataract and the recurrence of ulceration in herpetic eyes. Systemically administered corticosteroids and other immunosuppressive drugs may also cause adverse reactions. Tissue matching, although shown to be effective in corneal transplantation, is of little practical use at present because of major logistical problems.

Cyclosporin A, the subject of an article at page
148 , is a new and potent immunosuppressive agent which has aroused considerable interest in the field of transplantation. It has been shown to be effective in preventing allograft reactions in many organs and tissues in a variety of different animals and has been used clinically. It has a marked effect on the allograft response in the rabbit cornea. Its precise mode of action has not yet been determined, but it appears to inhibit the production of antibody by $T$ lymphocytes at the time of exposure to transplanted antigens. Although cyclosporin A lacks the adverse side reactions of other immunosuppressive drugs, it is not without toxic effects, most notably a significant incidence of lymphoma. However, it should be pointed out that lymphoma is a recognised complication of other immunosuppressive regimens.

The fact that cyclosporin $\mathrm{A}$ has been shown to inhibit the corneal allograft reaction in rabbits makes it a drug of considerable interest to ophthalmologists, though the present state of knowledge does not justify its use in clinical corneal transplantation. Clearly the effects of topical administration on corneal grafts need to be investigated. In the longer term the significance of cyclosporin A may prove to be that it heralds the advent of a new class of drugs having very specific effects on the allograft response. 